\title{
Directionally Selective Fractional Wavelet Transform Using a 2-D Non-Separable Unbalanced Lifting Structure
}

\author{
Furkan Keskin and A. Enis Çetin \\ Department of Electrical and Electronics Engineering, \\ Bilkent University, Bilkent, 06800, Ankara, Turkey \\ keskin@ee.bilkent.edu.tr, cetin@bilkent.edu.tr
}

\begin{abstract}
In this paper, we extend the recently introduced concept of fractional wavelet transform to obtain directional subbands of an image. Fractional wavelet decomposition is based on two-channel unbalanced lifting structures whereby it is possible to decompose a given discretetime signal $x[n]$ sampled with period $T$ into two sub-signals $x_{1}[n]$ and $x_{2}[n]$ whose average sampling periods are $p T$ and $q T$, respectively. Fractions $\mathrm{p}$ and $\mathrm{q}$ are rational numbers satisfying the condition: $1 / p+1 / q=1$. Filters used in the lifting structure are designed using the Lagrange interpolation formula. 2-d separable and non-separable extensions of the proposed fractional wavelet transform are developed. Using a non-separable unbalanced lifting structure, directional subimages for five different directions are obtained.
\end{abstract}

Keywords: Lifting, wavelet transform, multirate signal processing

\section{Introduction}

Lifting structures provide computationally efficient implementation of the wavelet transform [1-7] and they found applications in image and video coding and signal and image analysis applications $[8,9]$.

New unbalanced wavelet lifting structures producing directional decomposition of the input image are introduced in this article. In standard lifting structures the input signal is first decomposed into even and odd indexed samples using the lazy wavelet transform. In the recently proposed unbalanced lifting structure, a structure similar to the lazy filterbank forwards every $p^{\text {th }}$ sample of the original signal to the upper-branch and remaining $p-1$ samples out of $\mathrm{p}$ samples go to the lower branch [10]. Discrete-time update and prediction filters interconnect the upper and lower branches, respectively. Discrete-time filters operating in the lower sampling rates are designed using the Lagrange interpolation formula which is also used in many filterbank designs including our filterbanks, Smith-Barnwell and Daubechies filterbanks. In this way, two sub-signals with different sampling rates are obtained from the original signal. When the input signal has a sampling rate of $\mathrm{T}$ the upper- and lower- branches of the unbalanced lifting filterbank have sampling rates of $p T$ and $T p /(p-1)$, respectively. 
In standard balanced lifting the sampling periods of upper and lower branches are the same: $2 T$.

The unbalanced lifting decomposition can be easily generalized to other sampling strategies in which the upper-branch has a sampling rate of $p T$ and the lower-branch has a sampling rate of $q T$ with the property that

$$
\frac{1}{p}+\frac{1}{q}=1
$$

Perfect reconstruction can be easily achieved by changing the signs of the filters in the reconstruction part of the ordinary balanced lifting structures.

In Section 2, an example filterbank design with $p=3: 1$ and $q=3: 2$ is presented. In Section 3, 2-D separable filterbank design examples are presented. Non-separable 2-D extension of the unbalanced lifting structure resulting in directional subbands is developed in Section 4.

\section{Unbalanced Lazy Filterbank and Lifting Structures}

An unbalanced lazy filterbank for $p=3: 1$ and $q=3: 2$ is shown in Figure 2 . In the upper-branch a regular downsampling block by a factor of three is used. In the lower-branch the downsampling block for $q=3: 2$ is used. In Figure 2, the signal $x[n]$ is fed to the lazy filterbank and outputs of downsampling blocks are shown. Every $3^{\text {rd }}$ sample of the original signal goes to the upper-branch and remaining samples appear in the lower branch.

We describe the update and the prediction filter design for the unbalanced lifting structure in the following subsection.

\section{$2.1 \quad$ Update Filter}

In Figure 1 an unbalanced lifting structure is shown with downsampling ratios $3: 1$ and $3: 2$ in the upper and lower branches, respectively. Similar to the regular balanced lifting filterbank case, the upper branch sample $d$ can be estimated using the neighboring lower branch samples $b, c, e$, and $f$ and an estimate of the sample $d$ is given as follows:

$$
d^{\prime}=\frac{b+2 c+2 e+f}{6},
$$

which is the output of the update filter linking the lower branch to the upper branch. Since samples $c$ and $e$ are closer to the sample $d$ compared to $f$ and $b$ more weight is given to the samples $c$ and $e$. The sample $d$ and the output of the filter is linearly combined and the updated sample is obtained as follows

$$
\hat{d}=\frac{2 d^{\prime}}{3}+\frac{d}{3}
$$

Since the downsampling is by a factor of three in the upper-branch original signal must be filtered with a low-pass filter with a cut-off frequency of $\pi / 3$. We could 


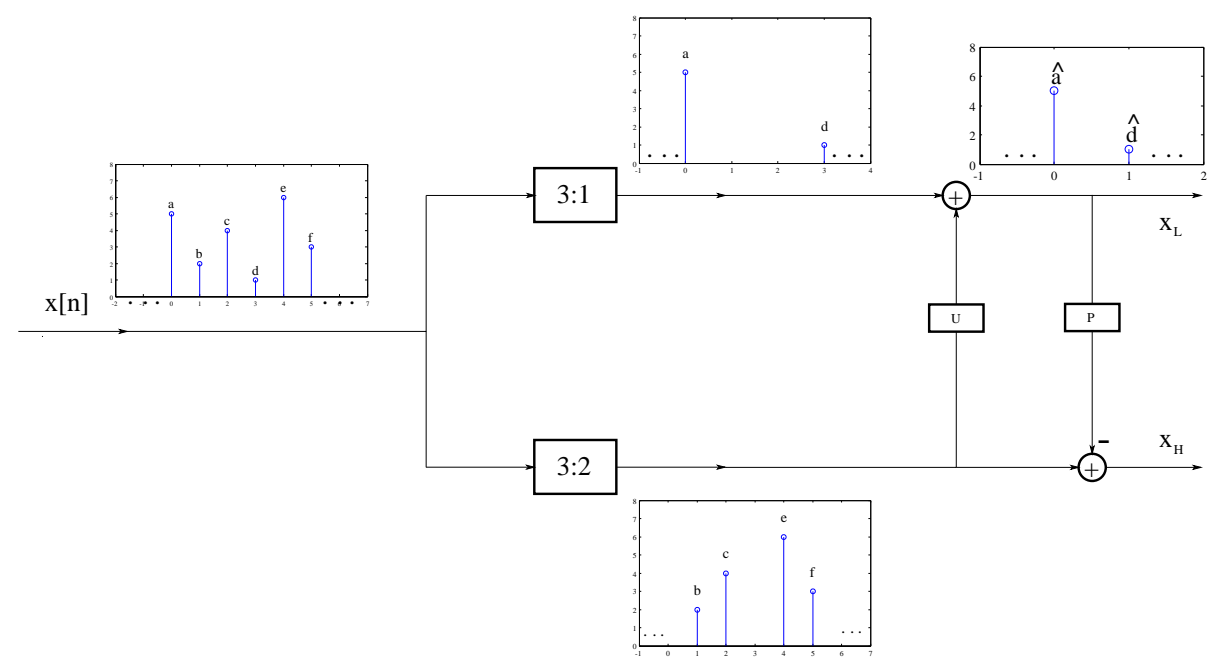

Fig. 1. Unbalanced lifting scheme with $p=3: 1$ and $q=3: 2$ downsampling ratios.

have used $\hat{d}=\frac{d^{\prime}}{2}+\frac{d}{2}$ but this does not produce as good frequency response as (3). Therefore, the upper branch output sample is given by

$$
\hat{d}=\frac{2 b}{18}+\frac{4 c}{18}+\frac{6 d}{18}+\frac{4 e}{18}+\frac{2 f}{18}
$$

The corresponding filter coefficients are given as follows

$$
h_{l}[n]=\left\{\frac{2}{18}, \frac{4}{18}, \frac{6}{18}, \frac{4}{18}, \frac{2}{18}\right\}
$$

The frequency response of this filter is plotted in [10]. The cut-off frequency of this filter is $\pi / 3$.

Other samples of the upper branch of the filterbank are smoothed in a similar manner. In Equation (5), each sample of the upper branch sub-signal is updated using four neighboring samples of the lower branch. It is possible to smooth the samples of the upper branch further by using more samples from the lower branch without effecting the perfect reconstruction capability of the filterbank. In this case, Lagrange interpolation formula or other interpolation methods can be used to determine the update filter coefficients. In general,

$$
x_{L}[n]=\frac{1}{9} x[3 n-2]+\frac{2}{9} x[3 n-1]+\frac{3}{9} x[3 n]+\frac{2}{9} x[3 n+1]+\frac{1}{9} x[3 n+2]
$$

where $x[n]$ is the original input signal to the filterbank.

\subsection{Prediction Filter}

Samples of the lower branch are estimated from the upper branch and difference is transmitted to the receiver. Lower branch samples can be predicted from the 
upper branch using the updated samples $\hat{a}, \hat{d}, \hat{g}, \ldots$ (see Figure 1 ). The prediction filter can be as simple as the identity operator selecting the nearest upper branch sample as an estimate of the lower branch sample:

$$
x_{H}[0]=b-\hat{a}, x_{H}[1]=c-\hat{d}, \ldots
$$

where the subscript $H$ indicates that $x_{H}[n]$ is a high-band sub-signal. Other samples of $x_{H}[n]$ can be determined in a similar manner. Although the above prediction strategy is very simple and computationally efficient, the above predictor is not a good estimator. We can use Lagrange interpolation and obtain:

$$
x_{H}[0]=b-(2 \hat{a}+\hat{d}) / 3, x_{H}[1]=c-(2 \hat{d}+\hat{a}) / 3, \ldots
$$

In general,

$$
x_{H}[n]= \begin{cases}x\left[\frac{3 n+2}{2}\right]-\frac{2 x_{U}\left[\frac{n}{2}\right]+x_{U}\left[\frac{n}{2}+1\right]}{3}, & \mathrm{n} \text { is even } \\ x\left[\frac{3 n+1}{2}\right]-\frac{2 x_{U}\left[\frac{n+1}{2}\right]+x_{U}\left[\frac{n-1}{2}\right]}{3}, & \mathrm{n} \text { is odd }\end{cases}
$$

where $x[n]$ is the original input signal to the filterbank.

To determine $x_{H}[0]$ the sample $b$ is estimated using the two nearest upper branch samples $\hat{a}$ and $\hat{d}$. Let $P(t)$ be the Lagrange interpolator based on the samples $\hat{a}$ and $\hat{d}$ :

$$
P(t)=\hat{a} \ell_{o}(t)+\hat{d} \ell_{1}(t)
$$

where $\ell_{i}(t)$ are the Lagrange basis polynomials. The function $P(t)$ is constructed using the input data pairs $(\hat{a}, 0)$ and $(\hat{d}, 3 T)$. Since the sample $b$ occurs at $t=T$, $\ell_{o}(T)=2 / 3$ and $\ell_{1}(T)=1 / 3$. Therefore the Lagrange interpolation gives more weight to the sample $\hat{a}$ because it is nearer to the sample $b$ compared to $\hat{d}$ to determine $x_{H}[0]$ in (8). Similarly, more weight is given to the sample $\hat{d}$ because it is nearer to the sample $c$ compared to $\hat{a}$ to determine $x_{H}[1]$ etc. As it can be seen from the above equations the predictor is a time varying filter. The predictor can even be an adaptive LMS-type filter as described in references $[4,11,12]$. The adaptive prediction tries to remove as much information as possible from the lower-branch using $x_{H}[n]$ samples. In this case the computational complexity is higher than the predictors in (7) and (8). Other samples of $x_{L}[n]$ can be also used by the predictor filter. In this case higher order Lagrange interpolation formula needs to be used $[4,13]$.

It is trivial to design the reconstruction filterbank as in regular lifting structures. At the reconstruction stage signs of filters $U$ and $P$ are changed and sub-signal samples are realigned to obtain the original signal $x[n]$.

Designing unbalanced lifting structures with different $p$ and $q$ values is also possible by following the abovementioned design strategy. For instance, an unbalanced lifting scheme with $p=3: 2$ and $q=3: 1$ can be constructed by determining the update and prediction filter coefficients according to distances between samples. It should be noted that low-pass filtered upper-branch signal is not uniformly sampled in this case. Hence, update and prediction filters are designed after transforming the upper branch samples to a uniform grid. Then, update and prediction operations can be carried out in a similar manner. 


\section{Extension for Two-Dimensional Signals}

In Figure 2, the separable filterbank structure for the unbalanced lifting wavelet transform with $p=3: 2$ and $q=3: 1$ is given. The input image is first downsampled in the horizontal direction. Then the update and prediction filters are applied to the downsampled images. The intermediate output signals $x_{L}$ and $x_{H}$ are then downsampled in the vertical direction and the update and prediction filters are applied again. As an example, the image given at Figure

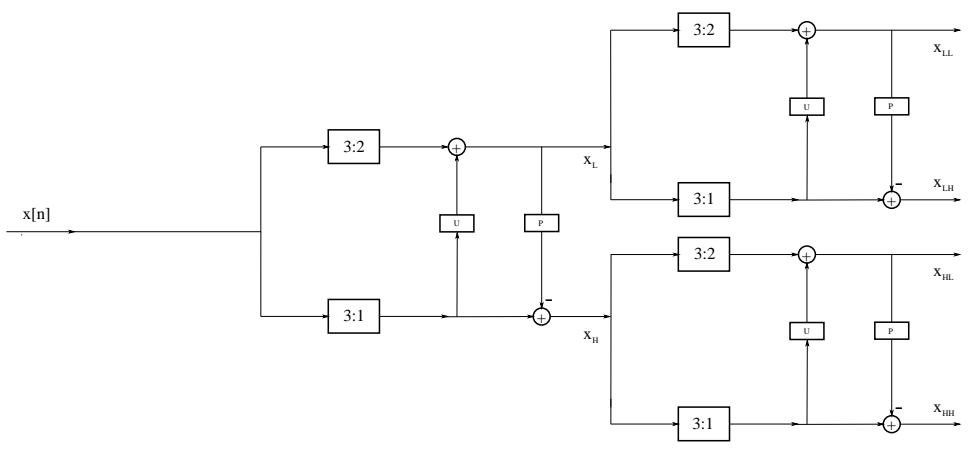

Fig. 2. Extension of the unbalanced lifting structure with $p=3: 2$ and $q=3: 1$ to $2-D$.

3 (left) is fractional wavelet transformed using the scheme presented in Figure 2. The transformed image is given at Figure 3 (right). As another example, the image given at Figure 4 is wavelet transformed in Figure 1 using the fractional wavelet transform with $p=3: 1$ and $q=3: 2$ which is a 2-D extended version of the filterbank described in Section 2.
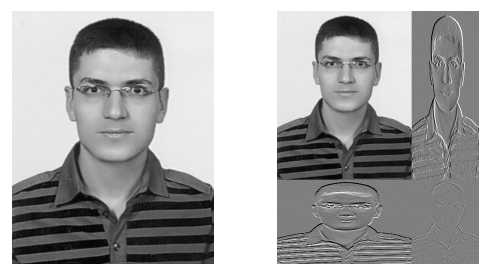

Fig. 3. Example image (left) and 2-D unbalanced lifting wavelet transformed image with $\mathrm{p}=3: 2$ and $\mathrm{q}=3: 1$ (right). Highband subimages are amplified by a factor of five. 


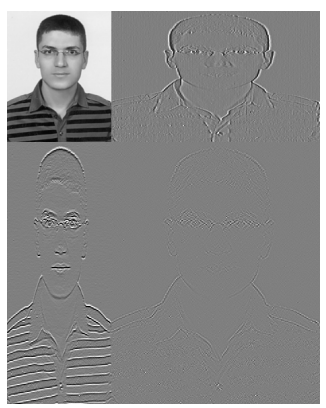

Fig. 4. 2-D unbalanced lifting wavelet transformed image with $p=3: 1$ and $q=3: 2$. Highband subimages are amplified by a factor of five.

\section{2-D Directionally Selective Non-Separable Unbalanced Filterbank Structure}

The 2-D non-separable extension of the unbalanced lifting wavelet transform is also possible. In Section 3, separable filterbank structure with $p=3: 2$ and $q=3: 1$ is proposed for two-dimensional signals. In this section, we design a non-separable lifting structure for 2-D signals with $p=9: 1$ and $q=9: 8$, where $p$ and $q$ denote spatial sampling rates. Figure 5 shows the non-separable structure having one upper branch and five lower branches, each corresponding to a prediction filter designed to reveal image edges in a specific direction.

In Figure 5, the upper branch has a downsampling ratio of $9: 1$ and the lower branch has a downsampling ratio of $9: 8$. First, a 2-D update filter is used to smooth the upper branch samples so that a low-resolution image $x_{L}\left[n_{1}, n_{2}\right]$ is obtained. Afterwards, 2-D prediction filters are used to obtain the directional subbands of the input image in five different directions, which are 0, 26.5, 45, 63.4 and 90 degrees with respect to the horizontal axis.

In Figure 5, pixels of an image are shown. The pixel marked $e$ can be estimated from the neighboring lower branch samples $a, b, c, d, f, g, h$, and $i$ as follows:

$$
e^{\prime}=\frac{a+c+g+i+\sqrt{2} b+\sqrt{2} f+\sqrt{2} h+\sqrt{2} d}{4 \sqrt{2}+4}
$$

The weights of the neighboring samples in estimating the sample $e$ are determined based on their geometric distance to the sample $e$. The estimate obtained in Equation (11) represents the output of the update filter and is linearly combined with the upper branch sample $e$ to obtain the updated sample corresponding to $e$ :

$$
\hat{e}=w_{1} e^{\prime}+w_{2} e
$$




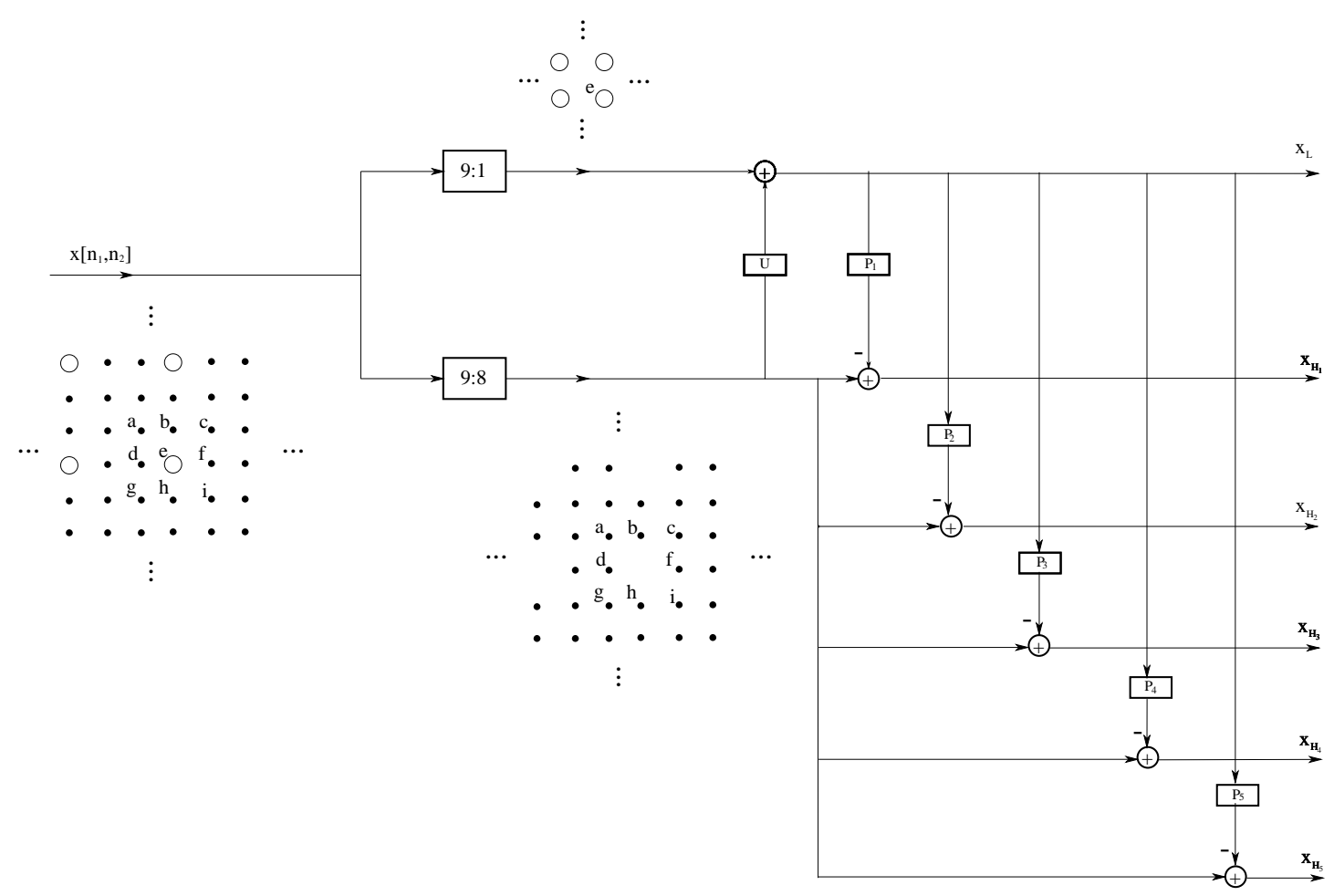

Fig. 5. 2-D non-separable extension of the unbalanced lifting structure with $p=9: 1$ and $q=9: 8$.

where $w_{1}$ and $w_{2}$ are weights which can be determined as in Section 4 such that the effective filter is a low-pass filter with cut-off frequency $w=\pi / 3$ in both horizontal and vertical directions.

Similar to the unbalanced lifting structure for 1-D signals, the original 2D signal must be filtered using a low-pass filter to avoid aliasing. The spatial downsampling ratio is $9: 1$ in the upper branch, which corresponds to a downsampling ratio of $3: 1 \mathrm{in}$ both horizontal and vertical directions. When $w_{1}=8 / 9$ and $w_{2}=1 / 9$, the upper branch output sample is computed as

$$
\hat{e}=\frac{2}{9(\sqrt{2}+1)}(a+c+g+i)+\frac{2 \sqrt{2}}{9(\sqrt{2}+1)}(b+f+h+d)+\frac{1}{9} e
$$

The coefficients of the filter that yields the upper branch samples from the original signal are given by

$$
h_{l}\left[n_{1}, n_{2}\right]=\left[\begin{array}{ccc}
\alpha & \beta & \alpha \\
\beta & \gamma & \beta \\
\alpha & \beta & \alpha
\end{array}\right]
$$


where $\alpha=\frac{2}{9(\sqrt{2}+1)}, \beta=\frac{2 \sqrt{2}}{9(\sqrt{2}+1)}$, and $\gamma=1 / 9$.

Frequency response of this symmetric filter is shown in Figure 6.

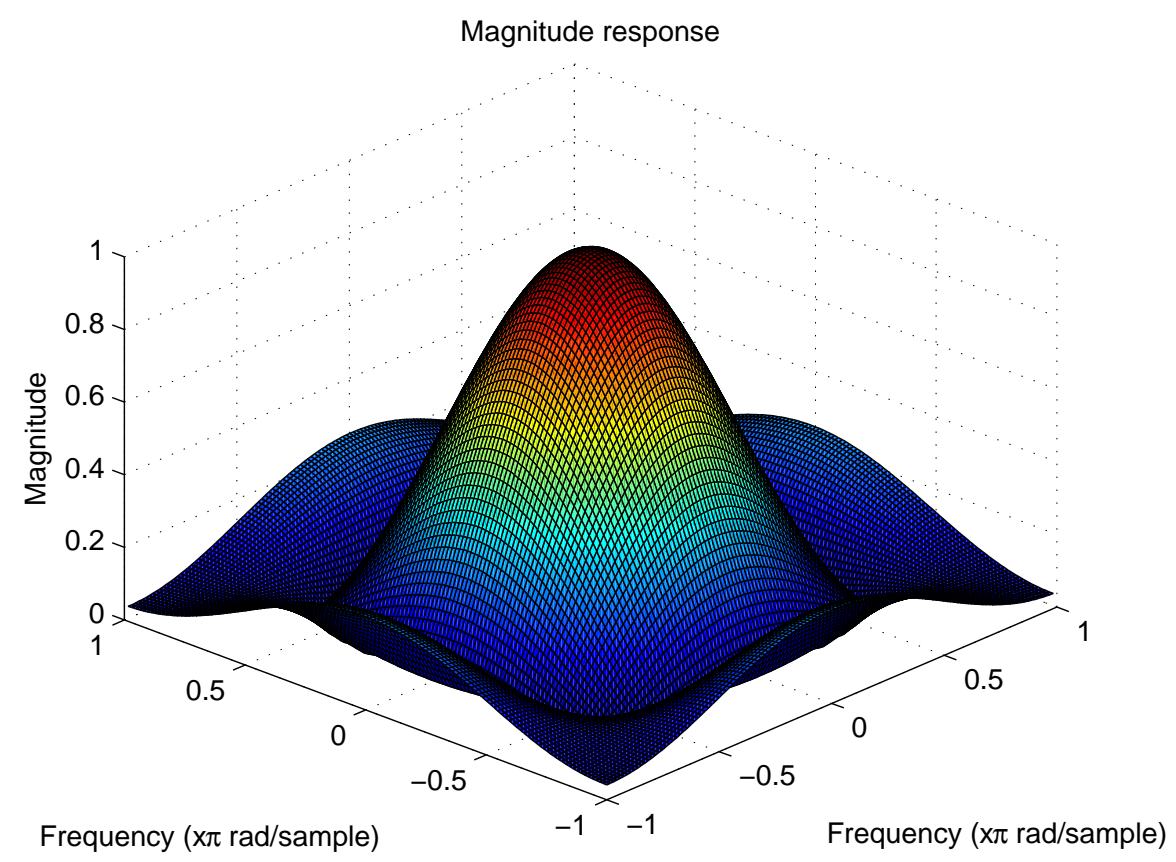

Fig. 6. Frequency response of the filter in Equation (14).

In general, the upper branch samples are given by

$$
\begin{gathered}
x_{L}\left[n_{1}, n_{2}\right]=\gamma x\left[3 n_{1}-2,3 n_{2}-2\right]+\alpha\left(x\left[3 n_{1}-3,3 n_{2}-3\right]+\right. \\
\left.x\left[3 n_{1}-3,3 n_{2}-1\right]+x\left[3 n_{1}-1,3 n_{2}-3\right]+x\left[3 n_{1}-1,3 n_{2}-1\right]\right)+ \\
\beta\left(x\left[3 n_{1}-3,3 n_{2}-2\right]+x\left[3 n_{1}-1,3 n_{2}-2\right]+\right. \\
\left.x\left[3 n_{1}-2,3 n_{2}-3\right]+x\left[3 n_{1}-2,3 n_{2}-1\right]\right)
\end{gathered}
$$

where $x\left[n_{1}, n_{2}\right]$ is the original input image to the filterbank.

Prediction filters $P_{1}, P_{2}, P_{3}, P_{4}$, and $P_{5}$ are designed to find estimates of the lower branch samples using the closest upper branch samples in five different directions, which are $0,26.5,45,63.4$ and 90 degrees, respectively, with respect to the horizontal axis. Figure 7 shows the upper branch samples $a_{i, j}$ and lower branch samples. The samples $y_{1}$ and $y_{2}$ are estimated by $P_{1}$ using the updated upper branch samples $a_{3,1}$ and $a_{3,2}$, and 0 degree high subband of the input 
image is constructed. The sample $v$ is estimated by $P_{2}$ using the upper branch samples $a_{3,1}$ and $a_{2,3}$, and the differences going into the second lower branch output channel make up the 26.5 degrees directional subband image. For the 45 degree diagonal subband, the samples $w_{1}$ and $w_{2}$ are predicted by using the upper branch samples $a_{3,1}$ and $a_{2,2} . P_{4}$ uses $a_{3,1}$ and $a_{1,2}$ samples to obtain the predictor for the sample $u$, resulting in 63.4 degrees directional subband image. Finally, the prediction filter $P_{5}$ produces estimates of the lower branch samples $z_{1}$ and $z_{2}$ using the upper branch samples $a_{3,1}$ and $a_{2,1}$, and the differences between the actual values and estimates form the 90 degrees directional subimage. Repeating these computations for each $3 \times 3$ subregion of a 2-D signal or an image as shown in Figure 7, we obtain directional subbands in a non-separable manner.

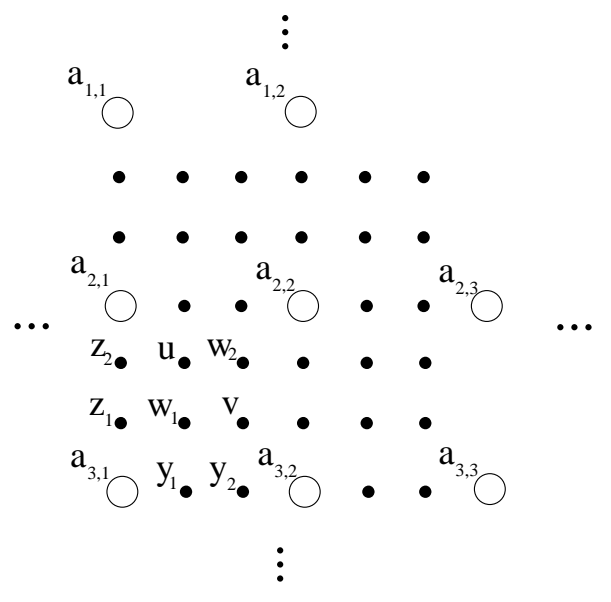

Fig. 7. Spatial organization of upper branch and lower branch samples.

To obtain the directional subimages by using the updated upper branch samples, the weights given to the upper branch samples are determined based on their geometric distance to the corresponding lower branch sample. Therefore, the closest neighboring upper branch sample has the weight $2 / 3$ and the other sample gets the weight $1 / 3$. For instance, for prediction of the sample $v, a_{3,1}$ is assigned $2 / 3$ weight and $a_{2,3}$ is assigned $1 / 3$ weight. Other lower branch samples can be predicted in a similar manner.

In general, directional subimages are given by the following equations:

$$
\begin{gathered}
x_{H_{1}}\left[n_{1}, n_{2}\right]=\left\{\begin{array}{l}
x\left[3 n_{1}-2, \frac{\left.3 n_{2}\right]}{2}\right]-\frac{2 x_{L}\left[n_{1}, \frac{n_{2}}{2}+1\right]+x_{L}\left[n_{1}, \frac{n_{2}}{2}\right]}{3}, \quad n_{2} \text { is even } \\
x\left[3 n_{1}-2, \frac{3 n_{2}+1}{2}\right]-\frac{2 x_{L}\left[n_{1}, \frac{n_{2}+1}{2}\right]+x_{L}\left[n_{1}, \frac{n_{2}+3}{2}\right]}{3}, n_{2} \text { is odd }
\end{array}\right. \\
x_{H_{2}}\left[n_{1}, n_{2}\right]=x\left[3 n_{1}, 3 n_{2}\right]-\frac{2 x_{L}\left[n_{1}+1, n_{2}\right]+x_{L}\left[n_{1}, n_{2}+2\right]}{3}
\end{gathered}
$$




$$
\begin{aligned}
& x_{H_{3}}\left[n_{1}, n_{2}\right]=\left\{\begin{array}{l}
x\left[3 n_{1}, \frac{3 n_{2}+1}{2}\right]-\frac{2 x_{L}\left[n_{1}+1, \frac{n_{2}+1}{2}\right]+x_{L}\left[n_{1}, \frac{n_{2}+3}{2}\right]}{3}, n_{2} \text { is odd } \\
x\left[3 n_{1}-1, \frac{3 n_{2}}{2}\right]-\frac{2 x_{L}\left[n_{1}, \frac{n_{2}}{2}+1\right]+x_{L}\left[n_{1}+1, \frac{n_{2}}{2}\right]}{3}, n_{2} \text { is even }
\end{array}\right. \\
& x_{H_{4}}\left[n_{1}, n_{2}\right]=x\left[3 n_{1}-1,3 n_{2}-1\right]-\frac{2 x_{L}\left[n_{1}+1, n_{2}\right]+x_{L}\left[n_{1}-1, n_{2}+1\right]}{3} \\
& x_{H_{5}}\left[n_{1}, n_{2}\right]= \begin{cases}x\left[\frac{3 n_{1}}{2}, 3 n_{2}-2\right]-\frac{2 x_{L}\left[\frac{n_{1}}{2}+1, n_{2}\right]+x_{L}\left[\frac{n_{1}}{2}, n_{2}\right]}{3}, & n_{1} \text { is even } \\
x\left[\frac{3 n_{1}+1}{2}, 3 n_{2}-2\right]-\frac{2 x_{L}\left[\frac{n_{1}+1}{2}, n_{2}\right]+x_{L}\left[\frac{n_{1}+3}{2}, n_{2}\right]}{3}, n_{1} \text { is odd }\end{cases}
\end{aligned}
$$

It is also possible to use the four closest neighboring upper branch samples in the desired direction instead of the two closest samples to estimate the lower branch sample. Then, the weights of the updated upper branch samples are assigned accordingly. More robust estimates of the lower branch samples can be achieved by adopting this prediction strategy. Other possibility is to use the adaptive filters in [6] and [7] for predicting the lower branch samples.

Figure 8 shows the directionally transformed image using the lifting scheme presented in Figure 5. Directional subband images reveal directional edges present in the input image. Non-separable unbalanced lifting structure provides directional edge information that can not be obtained by employing the separable filterbank structure. Figure 9 (left) shows another example image and Figure

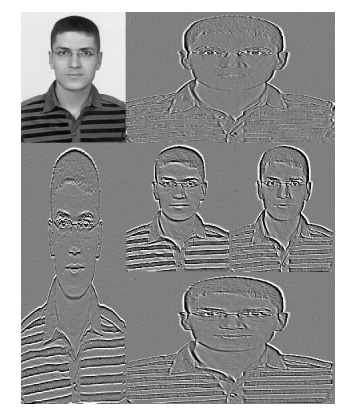

Fig. 8. 2-D unbalanced directional lifting wavelet transformed image with $\mathrm{p}=9: 1$ and $q=9: 8$. Highband subimages are amplified by a factor of five.

9 (right) shows the directional wavelet transformed subimages using the nonseparable unbalanced lifting scheme depicted in Figure 5. As observed from Figure 9, only the lines orthogonal to the direction in which the desired egdes are found are not highlighted in directional subband images. For instance, in 
63.4 degree directional subimage, the line that is in approximately 63.4 degree orientation with respect to the horizontal axis has the same gray value as the background, which indicates that there is almost no edge on this line in 63.4 degree direction with respect to the horizontal axis. Similarly, it is evident in 45 degree diagonal subband image that almost no directional edges are found on the line that makes an angle of 45 degree with the horizontal axis.

There are other possible lifting schemes than the one proposed in this paper for directionally selective fractional wavelet decomposition of images in a nonseparable manner. An alternative scheme is a 2-D non-separable unbalanced lifting structure where the spatial downsampling ratios are taken to be $p=16: 1$ and $q=16: 15$ for upper and lower branches, respectively. In this scheme directional subband images can be obtained for more than five directions by adding to the lifting structure several prediction filters tailored to desired directions. In this way, we have a more sophisticated directional wavelet decomposition with more detail about different angles while obtaining a lower resolution low-pass filtered subimage $x_{L}\left[n_{1}, n_{2}\right]$. Another possibility for an unbalanced lifting scheme is to employ a downsampling and prediction strategy such that directions ranging from 90 degree to 180 degree are also included in the directional decomposition of the input image.

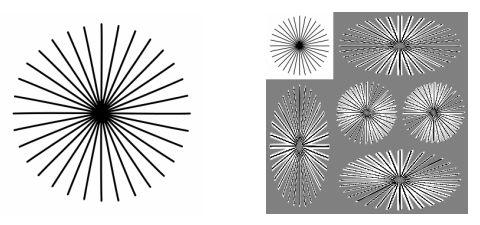

Fig. 9. Example image 2 (left) and 2-D unbalanced directional lifting wavelet transformed image with $\mathrm{p}=9: 1$ and $\mathrm{q}=9: 8$ (right). Highband subimages are amplified by a factor of five.

\section{Conclusion}

In this article, directionally selective fractional wavelet transform (FWT) methods are developed using unbalanced lifting structures. Sub-images have different average sampling rates.

2-D separable extensions carry out horizontal and vertical processing in different steps of the lifting transform. 2-D non-separable lifting structures provide 
unbalanced directional decomposition of the input image, leading to many different directional subbands. Image transformation examples are presented for both separable and non-separable cases. The FWT method can be easily extended to higher dimensions.

\section{References}

1. Mallat, S.: A Wavelet Tour of Signal Processing, Second Edition (Wavelet Analysis \& Its Applications). Academic Press (1999)

2. Sweldens, W.: The lifting scheme: A custom-design construction of biorthogonal wavelets. Applied and Computational Harmonic Analysis 3 (1996) 186 - 200

3. Daubechies, I., Sweldens, W.: Factoring wavelet transforms into lifting steps. Journal of Fourier Analysis and Applications 4 (1998) 247-269

4. Kim, C.W., Ansari, R., Cetin, A.E.: A class of linear-phase regular biorthogonal wavelets. In: Acoustics, Speech, and Signal Processing, IEEE International Conference on. Volume 4. (1992) $673-676$

5. Hampson, F.J., Pesquet, J.C.: A nonlinear subband decomposition with perfect reconstruction. In: Acoustics, Speech, and Signal Processing, IEEE International Conference on. Volume 3. (1996) $1523-1526$

6. Gerek, O.N., Cetin, A.E.: Adaptive polyphase subband decomposition structures for image compression. IEEE Transactions on Image Processing 9 (2000) 1649 $-1660$

7. Gerek, O.N., Cetin, A.E.: A 2-d orientation-adaptive prediction filter in lifting structures for image coding. IEEE Transactions on Image Processing 15 (2006) $106-111$

8. Le Pennec, E., Mallat, S.: Image compression with geometrical wavelets. In: Image Processing, International Conference on. Volume 1. (2000) 661-664

9. Claypoole, R.L., Davis, G.M., Sweldens, W., Baraniuk, R.G.: Nonlinear wavelet transforms for image coding via lifting. IEEE Transactions on Image Processing 12 (2003) $1449-1459$

10. Habiboglu, Y.H., K.K., Cetin, A.E.: Fractional wavelet transform using an unbalanced lifting structure. In: Independent Component Analyses, Wavelets, Neural Networks, Biosystems, and Nanoengineering IX, Proc. SPIE 8058. (2011)

11. Pesquet-Popescu, B., Bottreau, V.: Three-dimensional lifting schemes for motion compensated video compression. In: Acoustics, Speech, and Signal Processing, IEEE International Conference on. Volume 3. (2001) $1793-1796$

12. Piella, G., Pesquet-Popescu, B., Heijmans, H.: Adaptive update lifting with a decision rule based on derivative filters. IEEE Signal Processing Letters 9 (2002) $329-332$

13. Heller, P.: Lagrange $\mathrm{m}$-th band filters and the construction of smooth $\mathrm{m}$-band wavelets. In: Time-Frequency and Time-Scale Analysis, 1994. Proceedings of the IEEE-SP International Symposium on. (1994) $108-111$ 